VOL. 63 (2001) [279-298]

\title{
AN EXTENSION OF JENSEN'S FORM
}

\section{A. Sofo}

Arbitrary order forced difference-delay systems are considered, from which generated infinite sums of Binomial variation may be represented in closed form. It will be proved that the infinite sums may be expressed in a closed form which depends on dominant zeros of the polynomial characteristic functions. A connection between the infinite sum and generalised hypergeometric series will also be demonstrated.

\section{INTRODUCTION}

In a paper written in French and published in 1902, Jensen [3] substituted the functions

$$
\phi_{1}(z)=(z+1)^{n} \text { and } \phi_{2}(z)=(z+1)^{\beta}
$$

into the alternate form of Lagrange's inversion formula and obtained the identity

$$
\sum_{j=0}^{\infty}\left(\begin{array}{c}
n+j \beta \\
j
\end{array}\right) y^{j}=\frac{(z+1)^{n}}{1-\beta(z /(z+1))},
$$

where $y=z(z+1)^{-\beta}$.

This paper is concerned with the problem of generalising the identity

$$
\sum_{r=0}^{\infty}\left(\begin{array}{c}
n-a r \\
r
\end{array}\right) c^{r} b^{n-2 a r}=\frac{\xi_{0}^{n+1}}{(a+1) \xi_{0}-a b}
$$

where $\xi_{0}$ is the dominant zero, defined as the one with the greatest modulus, of the characteristic function

$$
g_{1}(z)=z^{a+1}-b z^{a}-c .
$$

On putting $c=\alpha b$ for $\alpha \in \mathbb{R}, a=-\beta, \alpha b^{\beta}=y$ and $z=\alpha \xi_{0}^{\beta}$, from $g_{1}\left(\xi_{0}\right)=0$ we have that $\xi_{0}=b(z+1)$ and substituting into (1.2), we obtain Jensen's identity (1.1). For

Received 5th June, 2000

I wish to acknowledge the useful discussions held with Associate Professor P. Cerone.

Copyright Clearance Centre, Inc. Serial-fee code: 0004-9727/01 \$A2.00+0.00. 
$\beta=0$, we note that (1.1) reduces to the Binomial Theorem. It may be shown, by the ratio test, that the left hand side of (1.2) converges in the region

$$
\left|\frac{c(a+1)^{a+1}}{b(a b)^{a}}\right|<1 .
$$

For $c=b$, Wilf [9] derived the partial sums of the left hand side of (1.2) and showed that it counts the number of words of $n$ letters over an alphabet of $b$ letters that do not contain the substring of a word of $a$ letters. For $a=1$, the partial sums of (1.2) reduce to well known sequences so that

$$
\begin{aligned}
h_{n} & =\sum_{r=0}^{[n / 2\rfloor}\left(\begin{array}{c}
n-r \\
r
\end{array}\right) c^{r} b^{n-2 r} \\
& =\frac{1}{\sqrt{b^{2}+4 a c}}\left(\left(\frac{b+\sqrt{b^{2}+4 a c}}{2}\right)^{n+1}-\left(\frac{b-\sqrt{b^{2}+4 a c}}{2}\right)^{n+1}\right) .
\end{aligned}
$$

The identity (1.3) is well known and $h_{n}$ may be identified with the Fibonacci, Jacobsthal, Pell, Fermat and Chebychev sequences. The sum (1.2) also has applications in discrete renewal theory processes, see Cohen [2], especially related to the bulk service variation of the $M / M^{(a)} / 1$ system.

\section{The SYSTEM}

Consider what we may describe as a generalised, or convoluted, Fibonacci sequence $f_{n}$, that satisfies

$$
\left\{\begin{array}{l}
\sum_{j=0}^{R}\left(\begin{array}{c}
R \\
R-j
\end{array}\right)(-b)^{R-j} \sum_{r=0}^{j}\left(\begin{array}{l}
j \\
r
\end{array}\right)(-b)^{j-r} f_{n+r-(R-j) a}=w_{n} ; n \geqslant a R \\
\sum_{r=0}^{R}\left(\begin{array}{l}
R \\
r
\end{array}\right)(-b)^{R-r} f_{n+r}=w_{n} ; n<a R
\end{array}\right.
$$

with $a$ and $R$ integers, $b$ real and where $w_{n}$ is a discrete forcing term. A method of analysing the solution of system (2.1) is by the use of $Z$ transform techniques. Without loss of generality, let $w_{n}=0, f_{R-1}=1$ and all other initial conditions of the system (2.1) be zero. If we now take the $Z$ transform of (2.1), use the two $Z$ transform properties,

$$
Z\left[f_{n+k}\right]=z^{k}\left[f(z)-\sum_{n=0}^{k-1} f_{n} z^{-n}\right]
$$

and

$$
Z\left[f_{n-k} U_{n-k}\right]=z^{-k} F(z)
$$


where $U_{n-k}$ is the discrete step function, we obtain

$$
F(z)\left\{\sum_{j=0}^{R}\left(\begin{array}{l}
R \\
j
\end{array}\right)(z-b)^{j}\left(-b z^{-a}\right)^{R-\jmath}\right\}=z
$$

and finally

$$
F(z)=\frac{z}{\left(z-b-b z^{-a}\right)^{R}}=\frac{z^{a R+1}}{(g(z))^{R}}
$$

where

$$
g(z)=z^{a+1}-b z^{a}-b
$$

In series form (2.2) may be rewritten as

$$
F(z)=\sum_{r=0}^{\infty}\left(\begin{array}{c}
R+r-1 \\
r
\end{array}\right) \frac{b^{r} z^{1-a r}}{(z-b)^{R+r}}
$$

which has an inverse

$$
f_{n}=\sum_{r=0}^{[(n+1-R) /(a+1)]}\left(\begin{array}{c}
R+r-1 \\
r
\end{array}\right)\left(\begin{array}{c}
n-a r \\
R+r-1
\end{array}\right) b^{n-a r-R+1}
$$

where $[x]$ represents the integer part of $x$. The inverse $Z$ transform of (2.2) may also be expressed as

$$
f_{n}=\frac{1}{2 \pi i} \oint_{C} z^{n}\left(\frac{F(z)}{z}\right) d z=\sum_{j=0}^{a} z^{n} \operatorname{Res}_{j}\left(\frac{F(z)}{z}\right),
$$

where $C$ is a smooth Jordan curve enclosing the singularities of (2.2) and $\operatorname{Res}_{j}$ is the residue of the poles of (2.2). The residue, $\operatorname{Res}_{j}$, depend on the zeros of the characteristic function in (2.3). Sofo and Cerone [5] have shown that the characteristic function, $g(z)$, with restriction

$$
\left|\frac{(a+1)^{a+1}}{(a b)^{a}}\right|<1
$$

has exactly $a+1$ distinct zeros $\xi_{j}, j=0,1,2,3, \ldots, a$, and therefore the singularities in (2.2) are all poles of order $R$. Thus a solution to (2.1) is

$$
f_{n}=\sum_{j=0}^{e} \sum_{\mu=0}^{R-1} Q_{R, \mu}\left(\xi_{j}\right)\left(\begin{array}{c}
n \\
R-1-\mu
\end{array}\right) \xi_{j}^{n-R+1+\mu}
$$

where

$$
\mu ! Q_{R, \mu}\left(\xi_{j}\right)=\lim _{x \rightarrow \xi,}\left[\frac{d^{\mu}}{d z^{\mu}}\left\{\left(z-\xi_{j}\right)^{R} \frac{F(z)}{z}\right\}\right]
$$


for each $j=0,1,2,3, \ldots, a$, with $F(z)$ being given by (2.2), and therefore we have that

$$
\begin{aligned}
\sum_{r=0}^{[(n+1-R) /(a+1)]}\left(\begin{array}{c}
R+r-1 \\
r
\end{array}\right)\left(\begin{array}{c}
n-a r \\
R+r-1
\end{array}\right) b^{n-a r-R+1} \\
=\sum_{j=0}^{a} \sum_{\mu=0}^{R-1} Q_{R, \mu}\left(\xi_{j}\right)\left(\begin{array}{c}
n \\
R-1-\mu
\end{array}\right) \xi_{j}^{n-R+1+\mu} .
\end{aligned}
$$

Recalling that the dominant zero $\xi_{0}$ of $g(z)$ in (2.3) is the one with the greatest modulus, we conjecture (and shortly prove) that

$$
\sum_{r=0}^{\infty} T_{r}(R, n, a, b)=\sum_{\mu=0}^{R-1} Q_{R, \mu}\left(\xi_{0}\right)\left(\begin{array}{c}
n \\
R-1-\mu
\end{array}\right) \xi_{0}^{n-R+1+\mu},
$$

where

$$
\mu ! Q_{R, \mu}\left(\xi_{0}\right)=\lim _{x \rightarrow \xi_{0}}\left[\frac{d^{\mu}}{d z^{\mu}}\left\{\left(z-\xi_{0}\right)^{R} \frac{F(z)}{z}\right\}\right], \quad \mu=0,1,2, \ldots, R-1 .
$$

$F(z)$ is given by $(2.2)$,

$$
T_{r}(R, n, a, b)=\left(\begin{array}{c}
r+R-1 \\
r
\end{array}\right)\left(\begin{array}{c}
n-a r \\
r+R-1
\end{array}\right) b^{n-a r-R+1}
$$

and the left hand side of (2.6) converges in the region described by (2.4). We also note, from (2.5) and (2.6), that

$$
\begin{aligned}
\sum_{r=0}^{[(n+1-R) /(a+1)]} T_{r}(R, n, a, b)+\sum_{r=[(n+2-R) /(a+1)]}^{\infty} T_{r}(R, n, a, b) \\
=\sum_{\mu=0}^{R-1} Q_{R, \mu}\left(\xi_{0}\right)\left(\begin{array}{c}
n \\
R-1-\mu
\end{array}\right) \xi_{0}^{n-R+1+\mu}
\end{aligned}
$$

and therefore

$$
\sum_{r=[(n+2-R) /(a+1)]}^{\infty} T_{r}(R, n, a, b)=-\sum_{j=1}^{a} \sum_{\mu=0}^{R-1} Q_{R, \mu}\left(\xi_{j}\right)\left(\begin{array}{c}
n \\
R-1-\mu
\end{array}\right) \xi_{j}^{n-R+1+\mu} .
$$

The conjecture (2.6) will be proved later. First we give a recurrence for the evaluation of (2.7) and a recurrence for (2.6).

\section{RECURRENCES}

A recurrence relation for the evaluation of $Q_{R, \mu}\left(\xi_{0}\right)$ in (2.7) is now given. 
LEMMA 1.

(3.1) $\quad(\mu+1) Q_{R, \mu+1}\left(\xi_{0}\right)=\frac{R\left(\xi_{0}-b\right)}{\xi_{0}^{2}} \sum_{k=0}^{\mu} \frac{(-1)^{k}(k+1)}{\xi_{0}^{k}}\left(\begin{array}{c}a+k+1 \\ a-1\end{array}\right) Q_{R+1, \mu-k}\left(\xi_{0}\right)$, $\mu=0,1,2, \ldots, R-1$, with $Q_{R, 0}\left(\xi_{0}\right)=\left(\xi_{0} /\left((a+1) \xi_{0}-a b\right)\right)^{R}$.

Proof: On putting $\mu=0$ into (2.7) we obtain the expression for $Q_{R, 0}\left(\xi_{0}\right)$. Also from the definition,

$$
\begin{aligned}
(\mu+1) ! Q_{R, \mu+1}\left(\xi_{0}\right) & =\lim _{x \rightarrow \xi_{0}}\left[\frac{d^{\mu+1}}{d z^{\mu+1}}\left\{\left(\frac{z^{a}\left(z-\xi_{0}\right)}{g(z)}\right)^{R}\right\}\right] \\
& =R \lim _{x \rightarrow \xi_{0}}\left[\frac{d^{\mu}}{d z^{\mu}}\left\{\left(\frac{z^{a}\left(z-\xi_{0}\right)}{g(z)}\right)^{R+1} \frac{h(z)}{\left(z-\xi_{0}\right)^{2}}\right\}\right]
\end{aligned}
$$

where

$$
h(z)=\frac{\left(a\left(z-\xi_{0}\right)+z\right) g(z)-z\left(z-\xi_{0}\right) g^{\prime}(z)}{z^{a+1}}
$$

and $g^{\prime}(z)=z^{a-1}((a+1) z-a b)$. It can be seen that $h\left(\xi_{0}\right)=0$ and $h^{\prime}\left(\xi_{0}\right)=0$, hence by expanding $h(z)$ in a Taylor series about $z=\xi_{0}$ we find that

$$
\frac{h(z)}{\left(z-\xi_{0}\right)^{2}}=\sum_{j=2}^{\infty} \frac{(-1)^{j}\left(\xi_{0}-b\right)(j-1)\left(z-\xi_{0}\right)^{j-2}}{\xi_{0}^{j}}\left(\begin{array}{c}
a+j-1 \\
a-1
\end{array}\right)=B(z) .
$$

\begin{tabular}{||c|c||}
\hline \hline$\mu$ & $\mu ! Q_{R, \mu}\left(\xi_{0}\right)$ \\
\hline 0 & $\frac{\xi_{0}^{R}}{A^{R}}$ \\
\hline 1 & $\frac{a R(A-b)}{2 A^{R+1}} \xi_{0}^{R-1}=\frac{a R(a+1)\left(\xi_{0}-b\right)}{2 A^{R+1}} \xi_{0}^{R-1}$ \\
\hline 2 & $\frac{a R \xi_{0}^{R-2}}{12 A^{R+2}}\left[A^{2}(3 a R-a-8)-2 b A(3 a R+a-4)+3(R+1) a b^{2}\right\}$ \\
\hline 3 & $\begin{array}{c}a R \xi_{0}^{R-3} \\
8 A^{R+3}\left[A^{3}\left\{a^{2} R(R-1)+2 a(1-4 R)+12\right\}\right. \\
+b A^{2}\left\{-a^{2} R(3 R+1)+2 a(8 R+3)-12\right\} \\
+b^{2} A\left\{a^{2}(3 R+2)(R+1)-8 a(R+1)\right\} \\
\end{array}$ \\
\hline
\end{tabular}

Table 1: The $Q$ values of the discrete case. 
We now have

$$
\begin{aligned}
(\mu+1) ! Q_{R, \mu+1}\left(\xi_{0}\right) & =R \lim _{z \rightarrow \xi_{0}}\left[\frac{d^{\mu}}{d z^{\mu}}\left\{\left(\frac{z^{a}\left(z-\xi_{0}\right)}{g(z)}\right)^{R+1} B(z)\right\}\right] \\
& =R \lim _{z \rightarrow \xi_{0}} \sum_{k=0}^{\mu}\left(\begin{array}{l}
\mu \\
k
\end{array}\right)\left(\frac{z^{a(R+1)}\left(z-\xi_{0}\right)^{R+1}}{g^{R+1}(z)}\right)^{(\mu-k)} B^{(k)}(z)
\end{aligned}
$$

where $B^{(k)}(z)=\frac{d^{k}}{d z^{k}} B(z)$, and

$$
\begin{aligned}
\lim _{z \rightarrow \xi_{0}}\left[\frac{d^{k}}{d z^{k}} B(z)\right] & =\lim _{z \rightarrow \xi_{0}}\left[\sum_{j=2}^{\infty} \frac{(-1)^{j}\left(\xi_{0}-b\right)(j-1)_{k}\left(z-\xi_{0}\right)^{j-2-k}}{\xi_{0}^{j}}\left(\begin{array}{c}
a+j-1 \\
a-1
\end{array}\right)\right] \\
& =\frac{(-1)^{k}\left(\xi_{0}-b\right)(k+1) !}{\xi_{0}^{k+2}}\left(\begin{array}{c}
a+k-1 \\
a-1
\end{array}\right), \quad k=0,1, \ldots, \mu
\end{aligned}
$$

where $(j-1)_{k}$ is Pochhammer's symbol. Hence we can write

$$
\begin{aligned}
& (\mu+1) ! Q_{R, \mu+1}\left(\xi_{0}\right) \\
& \quad=R \sum_{k=0}^{\mu}\left(\begin{array}{l}
\mu \\
k
\end{array}\right)(\mu-k) ! Q_{R+1, \mu-k}\left(\xi_{0}\right) \frac{(-1)^{k}\left(\xi_{0}-b\right)(k+1) !}{\xi_{0}^{k+2}}\left(\begin{array}{c}
a+k-1 \\
a-1
\end{array}\right)
\end{aligned}
$$

and upon simplification we obtain (3.1). Hence, Lemma 1 is proved.

We list some values of $Q_{R, \mu}\left(\xi_{0}\right)$ in Table 1, where for ease, $A=(a+1) \xi_{0}-a b$.

Using the values of $Q_{R, \mu}\left(\xi_{0}\right)$ in Table 1, some closed form expressions of (2.6) are listed in Table 2.

The following lemma gives a recurrence relation for the left hand side of identity (2.6).

LEMMA 2. Let

$$
S_{R}=\sum_{r=0}^{\infty} T_{r}(R, n, a, b)
$$

where $T_{r}(R, n, a, b)$ is given by (2.8). A recurrence relation for (3.2) is

$$
(a+1) b \frac{d}{d b} S_{R}-a b R S_{R+1}-(n+1-R) S_{R}=0 .
$$

PROOF:

$$
\frac{d}{d b} S_{R}=-\frac{a}{b} \sum_{r=0}^{\infty} r T_{r}(R, n, a, b)+\left(\frac{n+1-R}{b}\right) S_{R}
$$




\begin{tabular}{|c|c|}
\hline$R$ & closed form (2.6) \\
\hline 1 & $\xi_{0}^{n+1}\left[\frac{1}{A}\right]$ \\
\hline 2 & $\frac{\xi_{0}^{n+1}}{A^{2}}\left[n+\frac{a(a+1)\left(\xi_{0}-b\right)}{A}\right]$ \\
\hline 3 & $\frac{\xi_{0}^{n+1}}{A^{3}}\left[\left(\begin{array}{c}n \\
2\end{array}\right)+\frac{3 n a(A-b)}{2 A}+\frac{a\left(2(a-1) A^{2}-b A(5 a-2)+3 a b^{2}\right)}{2 A^{2}}\right.$ \\
\hline 4 & $\begin{array}{l}\frac{\xi_{0}^{n+1}}{A^{4}}\left[\left(\begin{array}{c}n \\
3\end{array}\right)+\frac{2 a(A-b)}{A}\left(\begin{array}{c}n \\
2\end{array}\right)\right. \\
+\frac{n a\left((11 a-8) A^{2}-2 b A(13 a-4)+15 a b^{2}\right)}{6 A^{2}} \\
+\frac{a}{12 A^{3}}\left(\begin{array}{c}A^{3}\left(12 a^{2}-30 a+12\right)-b A^{2}\left(52 a^{2}-70 a+12\right) \\
\left.\left.+b^{2} A a(70 a-40)-30 a^{2} b^{3}\right)\right]\end{array}\right.\end{array}$ \\
\hline
\end{tabular}

Table 2: Closed form of the discrete case.

and

$$
\begin{aligned}
S_{R+1} & =\sum_{r=0}^{\infty}\left(\begin{array}{c}
r+R \\
r
\end{array}\right)\left(\begin{array}{c}
n-a r \\
r+R
\end{array}\right) b^{n-a r-R} \\
& =-\frac{(a+1)}{b R} \sum_{r=0}^{\infty} r T_{r}(R, n, a, b)+\left(\frac{n+1-R}{b R}\right) S_{R} .
\end{aligned}
$$

From the left hand side of (3.3)

$$
\begin{aligned}
(a+1) b\left[-\frac{a}{b} \sum_{r=0}^{\infty}\right. & \left.r T_{r}(R, n, a, b)+\left(\frac{n+1-R}{b}\right) S_{R}\right] \\
& -a b R\left[-\frac{(a+1)}{b R} \sum_{r=0}^{\infty} r T_{r}(R, n, a, b)+\left(\frac{n+1-R}{b R}\right) S_{R}\right] \\
& -(n+1-R) S_{R}=0
\end{aligned}
$$

which is the right hand side of (3.3) and the proof is complete.

\section{Proof of the Conjecture}

The proof of conjecture (2.6) will involve an induction argument on the parameter $R$. For the basis, $R=1,(2.6)$ was proved by Sofo and Cerone [6]. Now we give an 
induction argument for the right hand side of (2.6).

$$
S_{R+1}=\frac{1}{a b R}\left\{(a+1) b \frac{d}{d b} S_{R}-(n+1-R) S_{R}\right\},
$$

also, $\frac{d}{d b} S_{R}=\left(\xi_{0}^{2} / b .4\right) \cdot \frac{d}{d \xi_{0}} S_{R}$ and

$$
\frac{d}{d b} S_{R}=\frac{\xi_{0}^{2}}{b A} \sum_{\mu=0}^{R-1}\left(\begin{array}{c}
n \\
R-1-\mu
\end{array}\right) \xi_{0}^{n-R+1+\mu} \times\left\{\frac{d}{d \xi_{0}} Q_{R, \mu}\left(\xi_{0}\right)+\left(\frac{n+1+\mu-R}{\xi_{0}}\right) Q_{R, \mu}\left(\xi_{0}\right)\right\} .
$$

Substituting into the right hand side of (4.1), we have

$$
\begin{aligned}
& S_{R+1}=\frac{1}{a b R}\left\{\frac { ( a + 1 ) b \xi _ { 0 } ^ { 2 } } { b A } \sum _ { \mu = 0 } ^ { R - 1 } ( \begin{array} { c } 
{ n } \\
{ R - 1 - \mu }
\end{array} ) \xi _ { 0 } ^ { n - R + 1 + \mu } \left(\frac{d}{d \xi_{0}} Q_{R, \mu}\left(\xi_{0}\right)\right.\right. \\
& \left.+\left(\frac{n+1+\mu-R}{\xi_{0}}\right) Q_{R, \mu}\left(\xi_{0}\right)\right) \\
& \left.-(n+1-R) \sum_{\mu=0}^{R-1}\left(\begin{array}{c}
n \\
R-1-\mu
\end{array}\right) \xi_{0}^{n-R+1+\mu} Q_{R, \mu}\left(\xi_{0}\right)\right\} \\
& =\frac{1}{a b R A} \sum_{\mu=0}^{R-1}\left(\begin{array}{c}
n \\
R-1-\mu
\end{array}\right) \xi_{0}^{n-R+1+\mu}\left\{(A+a b) \xi_{0} \frac{d}{d \xi_{0}} Q_{R, \mu}\left(\xi_{0}\right)\right. \\
& \left.+\mu A Q_{R, \mu}\left(\xi_{0}\right)+a b(n+1+\mu-R) Q_{R, \mu}\left(\xi_{0}\right)\right\} \\
& =\frac{1}{a b R A}\left\{\sum_{\mu=0}^{R-1} a b(R-\mu) \xi_{0}\left(\begin{array}{c}
n \\
R-\mu
\end{array}\right) \xi_{0}^{n-R+\mu} Q_{R, \mu}\left(\xi_{0}\right)\right. \\
& \left.+\sum_{\mu=0}^{R-1}\left(\begin{array}{c}
n \\
R-1-\mu
\end{array}\right) \xi_{0}^{n-R+1+\mu}\left((A+a b) \xi_{0} \frac{d}{d \xi_{0}} Q_{R, \mu}\left(\xi_{0}\right)+\mu A Q_{R, \mu}\left(\xi_{0}\right)\right)\right\} \\
& =\frac{1}{a b R A}\left\{a b R \xi_{0}\left(\begin{array}{l}
n \\
R
\end{array}\right) \xi_{0}^{n-R} Q_{R, 0}\left(\xi_{0}\right)\right. \\
& +\sum_{\mu=1}^{R-1} a b(R-\mu)\left(\begin{array}{c}
n \\
R-\mu
\end{array}\right) \xi_{0}^{n-R+1+\mu} Q_{R, \mu}\left(\xi_{0}\right) \\
& \left.+\sum_{\mu=0}^{R-1}\left(\begin{array}{c}
n \\
R-1-\mu
\end{array}\right) \xi_{0}^{n-R+1+\mu}\left((A+a b) \xi_{0} \frac{d}{d \xi_{0}} Q_{R, \mu}\left(\xi_{0}\right)+\mu A Q_{R, \mu}\left(\xi_{0}\right)\right)\right\} \text {. }
\end{aligned}
$$


In the second sum rename $\mu^{*}=\mu+1$ (and let $\mu^{*}=\mu$ ), so that we have

$$
\begin{gathered}
\left(\begin{array}{l}
n \\
R
\end{array}\right) \frac{\xi_{0}}{A} \xi_{0}^{n-R} Q_{R, 0}\left(\xi_{0}\right)+\frac{1}{a b R A}\left\{\sum_{\mu=1}^{R-1} a b(R-\mu)\left(\begin{array}{c}
n \\
R-\mu
\end{array}\right) \xi_{0}^{n-R+1+\mu} Q_{R, \mu}\left(\xi_{0}\right)\right. \\
\left.\quad+\sum_{\mu=1}^{R-1}\left(\begin{array}{c}
n \\
R-\mu
\end{array}\right) \xi_{0}^{n-R+\mu}\left((A+a b) \xi_{0} \frac{d}{d \xi_{0}} Q_{R, \mu-1}\left(\xi_{0}\right)+(\mu-1) A Q_{R, \mu-1}\left(\xi_{0}\right)\right)\right\} \\
=\left(\begin{array}{c}
n \\
R
\end{array}\right) \xi_{0}^{n-R} Q_{1,0}\left(\xi_{0}\right) Q_{R, 0}\left(\xi_{0}\right) \\
\quad+\frac{1}{a b R A} \sum_{\mu=1}^{R-1}\left(\begin{array}{c}
n \\
R-\mu
\end{array}\right) \xi_{0}^{n-R+\mu}\left\{(A+a b) \xi_{0} \frac{d}{d \xi_{0}} Q_{R, \mu-1}\left(\xi_{0}\right)\right. \\
\left.\quad+a b(R-\mu) \xi_{0} Q_{R, \mu}\left(\xi_{0}\right)+(\mu-1) A Q_{R, \mu-1}\left(\xi_{0}\right)\right\} \\
=\left(\begin{array}{c}
n \\
R
\end{array}\right) \xi_{0}^{n-R} Q_{R+1,0}\left(\xi_{0}\right)+\frac{1}{a b R A} \sum_{\mu=1}^{R-1}\left(\begin{array}{c}
n-\mu \\
R-\mu
\end{array}\right) \xi_{0}^{n-R+\mu}\left\{a b(R-\mu) \xi_{0} Q_{R, \mu}\left(\xi_{0}\right)\right. \\
\left.\quad+(A+a b) \xi_{0} \frac{d}{d \xi_{0}} Q_{R, \mu-1}\left(\xi_{0}\right)+(\mu-1) A Q_{R, \mu-1}\left(\xi_{0}\right)\right\} .
\end{gathered}
$$

Using the relationship

$$
\begin{aligned}
a b R A Q_{R+1, \mu}\left(\xi_{0}\right)=a b(R-\mu) \xi_{0} Q_{R, \mu}\left(\xi_{0}\right) & \\
& +(\mu-1) A Q_{R, \mu-1}\left(\xi_{0}\right)+(A+a b) \xi_{0} \frac{d}{d \xi_{0}} Q_{R, \mu-1}\left(\xi_{0}\right)
\end{aligned}
$$

proved by Sofo and Cerone [5], (the style of proof may also be seen in [8]), reduces the previous line to

$$
\begin{aligned}
\left(\begin{array}{l}
n \\
R
\end{array}\right) \xi_{0}^{n-R} Q_{R+1,0}\left(\xi_{0}\right)+\sum_{\mu=1}^{R-1}\left(\begin{array}{c}
n \\
R-\mu
\end{array}\right) \xi_{0}^{n-R+\mu} Q_{R+1, \mu}\left(\xi_{0}\right) & \\
& =\sum_{\mu=0}^{R}\left(\begin{array}{c}
n \\
R-\mu
\end{array}\right) \xi_{0}^{n-R+\mu} Q_{R+1, \mu}\left(\xi_{0}\right),
\end{aligned}
$$

which completes the proof of the conjecture.

The degenerate case, for $a=0$, of identity (2.6) can be noted. Firstly, $\xi_{0}=2 b$ and $\mu ! Q_{R, \mu}(2 b)=1$ for $\mu=0$ and zero otherwise, hence (2.6) reduces to

$$
\sum_{r=0}^{n+1-R}\left(\begin{array}{c}
n+1-R \\
r
\end{array}\right)=2^{n+1-R}
$$

which is not Gosper summable, as defined by Petkovšek et al. [4]. However by the $W Z$ pairs method [4] a rational certificate function is

$$
V(n, r)=\frac{r}{2(r-n-2+R)}
$$




\section{HyPERGEOMETRIC FUNCTIONS}

Let $T_{r}(R, n, a, b)$ be defined by $(2.8)$ and $T_{0}(R, n, a, b)=\left(\begin{array}{c}n \\ R-1\end{array}\right) b^{n+1-R}$. The ratio of consecutive terms

$$
\frac{T_{r+1}(R, n, a, b)}{T_{r}(R, n, a, b)}=\frac{\prod_{j=0}^{a}(r+(j+R-n-1) /(a+1))}{(r+1) \prod_{j=0}^{a-1}(r+((j-n) / a))} s(a, b)
$$

is a rational function in $r$ and therefore the series $S_{R}(n, a, b)$ of (3.2) may be expressed as a generalised hypergeometric function

$$
\text { (5.1) } \begin{array}{r}
T_{0 a+1} F_{a}\left[\begin{array}{c}
\frac{R-n-1}{a+1}, \frac{R-n}{a+1}, \frac{R-n+1}{a+1}, \ldots, \frac{R+a-n-1}{a+1} \mid s(a, b) \\
-\frac{n}{a}, \frac{1-n}{a}, \frac{2-n}{a}, \ldots, \frac{a-1-n}{a}
\end{array}\right] \\
=T_{0} \sum_{k=0}^{\infty} \frac{((R-n-1) /(a+1))_{k}((R-n) /(a+1))_{k}((R-n+1) /(a+1))_{k}}{(-(n / a))_{k}((1-n) / a)_{k}((2-n) / a)_{k}} \\
\frac{\ldots((R+a-n-1) /(a+1))_{k}}{\ldots((a-1-n) / a)_{k}} \cdot \frac{s^{k}(a, b)}{k !}
\end{array}
$$

where $(x)_{m}$ is Pochhammer's symbol and

$$
s(a, b)=-\frac{(a+1)^{a+1}}{(a b)^{a}} .
$$

Some particular cases of (5.1) are worthy of mention, since it may be shown that (5.1) reduces to known hypergeometric functions. For $a=1$ and $\alpha=-n \in \mathbb{R} \backslash \mathrm{J}^{-}$then

$$
T_{02} F_{1}\left[\frac{R+\alpha-1}{2}, \frac{R+\alpha}{2} \mid s(1, b)\right]=T_{0}\left[1+\sum_{k=1}^{\infty} \frac{(-1)^{k}}{k ! b^{k}} \cdot \frac{\prod_{j=0}^{2 k-1}(R+\alpha-1+j)}{\prod_{j=0}^{k-1}(\alpha+j)}\right] .
$$

The Gauss hypergeometric series (5.2) may also be written as

$$
\frac{T_{0}}{B((R+\alpha) / 2,(\alpha-R) / 2)} \int_{t=0}^{1}(1-t)^{(\alpha-R-2) / 2} t^{(R+\alpha-2) / 2}(1-s(1, b) t)^{(1-R-\alpha) / 2} d t
$$

which is valid for $|s(1, b)|<1$, where $B(x, y)$ is the Beta function. The difference in the two top terms of the hypergeometric function (5.2) is one-half, hence there exists a quadratic transformation, see [1], connected with the Legendre function, $P_{\nu}^{\mu}$. By using the identity of Abramowitz and Stegun [1, p. 562], we may write (5.2) as

$$
T_{0} 2^{\alpha-1} \Gamma(\alpha)\{-s(1, b)\}^{(1-\alpha) / 2}\{1-s(1, b)\}^{-R / 2} P_{R-1}^{1-\alpha}\left\{\{1-s(1, b)\}^{-1 / 2}\right\},
$$


where $s(1, b) \in(-\infty, 0)$ and $\Gamma(x)$ is the Gamma function. We may write the identity (2.6) as

$$
\begin{aligned}
\sum_{r=0}^{\infty}\left(\begin{array}{c}
R+r-1 \\
r
\end{array}\right)\left(\begin{array}{c}
-\alpha-r \\
R+r-1
\end{array}\right) b^{-\alpha-r-R+1} & \\
= & \sum_{\mu=0}^{R-1} Q_{R, \mu}\left(\xi_{0}\right)\left(\begin{array}{c}
-\alpha \\
R-1-\mu
\end{array}\right) \xi_{0}^{n-R+1+\mu} \\
& =\frac{T_{0} \Gamma(\alpha)}{2^{1-\alpha}}\left\{\frac{4}{b}\right\}^{(1-\alpha) / 2}\left\{1+\frac{4}{b}\right\}^{-(R / 2)} P_{R-1}^{1-\alpha}\left\{\left\{1+\frac{4}{b}\right\}^{-1 / 2}\right\}
\end{aligned}
$$

for $b>4$, where $2 \xi_{0}=b+\sqrt{b^{2}+4 b}$ and $Q_{R, \mu}\left(\xi_{0}\right)$ is defined by (2.7). Other specific cases of (5.2) are as follows.

(i) For $b=4, s(1,4)=-1, \alpha=3 / 2$, using (5.2) and Abramowitz and Stegun $[1$, p. 557]

$$
\begin{aligned}
T_{02} F_{1} & {\left[\begin{array}{c|c}
\frac{2 R+1}{4}, \frac{2 R+3}{4} \mid & -1 \\
\frac{3}{2} & \mid
\end{array}\right]=\frac{T_{0} 2^{-(2 R+1) / 4} \Gamma(3 / 2) \sqrt{\pi}}{(2 R-1) / 4} \times } \\
& \left\{\frac{1}{\Gamma((R / 4)+(1 / 8)) \Gamma((7 / 8)-(R / 4))}-\frac{1}{\Gamma((R / 4)+(5 / 8)) \Gamma((3 / 8)-(R / 4))}\right\} .
\end{aligned}
$$

Since the parameters in the hypergeometric function give

$$
\frac{2 R+1}{4}-\frac{2 R+3}{4}+\frac{3}{2}=1
$$

from Kummer's identity we have

$$
\begin{aligned}
& \sum_{r=0}^{\infty}\left(\begin{array}{c}
R+r-1 \\
r
\end{array}\right)\left(\begin{array}{c}
-\frac{3}{2}-r \\
R+r-1
\end{array}\right) 4^{-(1 / 2)-r-R} \\
& \quad=\sum_{\mu=0}^{R-1} Q_{R, \mu}\left(\xi_{0}\right)\left(\begin{array}{c}
-\frac{3}{2} \\
R-1-\mu
\end{array}\right) \xi_{0}^{\mu-R-(1 / 2)} \\
& \quad=T_{02} F_{1}\left[\begin{array}{c}
\frac{2 R+1}{4}, \frac{2 R+3}{4} \\
\frac{3}{2}
\end{array} \mid-1\right]=\frac{T_{0} \Gamma(3 / 2) \Gamma((R / 4)+(11 / 8))}{\Gamma((R / 2)+(7 / 4)) \Gamma((9 / 8)-(R / 4))} .
\end{aligned}
$$

Here the dominant zero is $\xi_{0}=2(1+\sqrt{2})$ and some values of the infinite sum are

\begin{tabular}{||c|c|c|c||}
\hline \hline$R=1$ & $R=2$ & $R=3$ & $R=4$ \\
\hline$\frac{1}{8(1+\sqrt{2})^{1 / 2}}$ & $\frac{-(2+\sqrt{2})}{2\left(4^{3}\right)(1+\sqrt{2})^{1 / 2}}$ & $\frac{3(2+\sqrt{2})}{2\left(4^{5}\right) \sqrt{2}(1+\sqrt{2})^{1 / 2}}$ & $\frac{-5}{4^{7} \sqrt{2}(1+\sqrt{2})^{1 / 2}}$ \\
\hline
\end{tabular}


(ii) For $b=4, s(1,4)=-1, \alpha=1 / 2$, from Abramowitz and Stegun [1, p. 557] we have

$$
\begin{aligned}
& \sum_{r=0}^{\infty}\left(\begin{array}{c}
R+r-1 \\
r
\end{array}\right)\left(\begin{array}{c}
-\frac{1}{2}-r \\
R+r-1
\end{array}\right) 4^{(1 / 2)-r-R} \\
& \quad=\sum_{\mu=0}^{R-1} Q_{R, \mu}\left(\xi_{0}\right)\left(\begin{array}{c}
-\frac{1}{2} \\
R-1-\mu
\end{array}\right) \xi_{0}^{\mu-R+(1 / 2)} \\
& \quad=T_{02} F_{1}\left[\begin{array}{c}
\frac{2 R-1}{4}, \frac{2 R+1}{4} \\
\frac{1}{2}
\end{array} \mid-1\right]=\frac{T_{0} \pi 2^{(1-2 R) / 2}}{\Gamma((R / 2)+(3 / 8)) \Gamma((5 / 8)-(R / 4))}
\end{aligned}
$$

and some values of the infinite sum are

\begin{tabular}{||c|c|c|c||}
\hline$R=1$ & $R=2$ & $R=3$ & $R=4$ \\
\hline$\frac{(1+\sqrt{2})^{1 / 2}}{4}$ & $\frac{(1+\sqrt{2})^{1 / 2}(\sqrt{2}-2)}{4^{3}}$ & $\frac{3 \sqrt{2}(1+\sqrt{2})^{1 / 2}(\sqrt{2}-2)}{2\left(4^{5}\right)}$ & $\frac{5 \sqrt{2}(1+\sqrt{2})^{1 / 2}}{4^{7}}$ \\
\hline
\end{tabular}

\section{ForCing TERMS}

If in the dynamical system (2.1) we consider forcing terms of the type

$$
w_{n}=\left(\begin{array}{c}
n \\
m+R-1
\end{array}\right) b^{n+1-R-m}
$$

for $m$ integer, and all initial conditions at rest, (other forcing terms may also be considered) then the result is the identity

$$
\begin{aligned}
& \sum_{r=0}^{\infty}\left(\begin{array}{c}
R+r-1 \\
r
\end{array}\right)\left(\begin{array}{c}
n-a r \\
r+R+m-1
\end{array}\right) b^{n-a r-R-m+1} \\
&=\sum_{\mu=0}^{R-1} Q_{R, \mu}\left(\xi_{0}\right)\left(\begin{array}{c}
n \\
R-1-\mu
\end{array}\right) \xi_{0}^{n-R+1+\mu} \\
& \quad+\sum_{\nu=0}^{m-1} P_{m, \nu}(b)\left(\begin{array}{c}
n \\
m-1-\nu
\end{array}\right) b^{n-m+1+\nu}
\end{aligned}
$$

where

$$
\begin{aligned}
& \mu ! Q_{R, \mu}\left(\xi_{0}\right)=\lim _{x \rightarrow \xi_{0}}\left[\frac{d^{\mu}}{d z^{\mu}}\left\{\left(z-\xi_{0}\right)^{R} \frac{F(z)}{z}\right\}\right], \\
& \nu ! P_{m, \nu}\left(\xi_{0}\right)=\lim _{z \rightarrow b}\left[\frac{d^{\nu}}{d z^{\nu}}\left\{(z-b)^{m} \frac{F(z)}{z}\right\}\right]
\end{aligned}
$$


and

$$
F(z)=\frac{z^{a R+1}}{(z-b)^{m}\left(z^{a+1}-b z^{a}-b\right)^{R}},
$$

where $\xi_{0}$ is the dominant zero of (2.3). If $R=2$ and $m=1$, then

$$
\begin{aligned}
\sum_{r=0}^{\infty}\left(\begin{array}{c}
r+1 \\
r
\end{array}\right)\left(\begin{array}{c}
n-a r \\
r+2
\end{array}\right)\left(\frac{1}{b^{a}}\right)^{r} & \\
& =b^{2 a}+\frac{b^{2-n} \xi_{0}^{1+n}\left(n\left((a+1) \xi_{0}-a b\right)+a(a+1)\left(\xi_{0}-b\right)\right)}{\left(\xi_{0}-b\right)\left((a+1) \xi_{0}-a b\right)} .
\end{aligned}
$$

For the degenerate case $a=0$, we obtain the interesting Binomial convolution identity

$$
\begin{gathered}
\sum_{r=0}^{n+1-m-R}\left(\begin{array}{c}
R+r-1 \\
r
\end{array}\right)\left(\begin{array}{c}
n \\
r+R+m-1
\end{array}\right) \\
=\sum_{\nu=0}^{m-1}(-1)^{R}\left(\begin{array}{c}
R+\nu-1 \\
\nu
\end{array}\right)\left(\begin{array}{c}
n \\
m-1-\nu
\end{array}\right) \\
\quad+\sum_{\mu=0}^{R-1}(-1)^{\mu}\left(\begin{array}{c}
m+\mu-1 \\
\mu
\end{array}\right)\left(\begin{array}{c}
n \\
R-1-\mu
\end{array}\right) 2^{n-R+1+\mu} \\
=\frac{{ }_{2} F_{1}\left[\begin{array}{c}
R, R+m-n-1 \\
R+m
\end{array} \mid-1\right]}{(n+1) B(R+m, n+2-R-m)},
\end{gathered}
$$

which for specific values of $m$ and $R$ may be certified by the $W Z$ pairs method of Wilf and Zeilberger (where $B(x, y)$ is the classical Beta function).

\section{Multiple Dominant Zeros}

In this section we shall generalise identity (2.6). We shall prove that a generated infinite series may be represented in a closed form that depends on the $k$ dominant zeros of an associated polynomial characteristic function. Consider the delay system

$$
\begin{cases}\sum_{j=0}^{k}\left(\begin{array}{l}
k \\
j
\end{array}\right)(-b)^{k-j} f_{n+j}+c f_{n-a k}=w_{n} ; & n \geqslant a k \\
\sum_{j=0}^{k}\left(\begin{array}{l}
k \\
j
\end{array}\right)(-b)^{k-j} f_{n+j}=w_{n} ; & n<a k\end{cases}
$$

with $c+b^{k}=0$ and all initial conditions at rest, except for $f_{k-1}=1$. Again, as previously, we set $w_{n}=0$ and take the $Z$ transform of (7.1) such that

$$
F(z)=\frac{z}{(z-b)^{k}-\left(b z^{-a}\right)^{k}}=\frac{z^{a k+1}}{\left(z^{a}(z-b)\right)^{k}-b^{k}} .
$$


Upon inversion,

$$
f_{n}=\sum_{r=0}^{[(n-k+1) /(a+1) k\}}\left(\begin{array}{c}
n-a k r \\
k r+k-1
\end{array}\right) b^{n-a k r-k+1}
$$

Also, from (7.2) we may write

$$
f_{n}=\frac{1}{2 \pi i} \oint_{C} z^{n}\left(\frac{F(z)}{z}\right) d z=\sum_{j=0}^{k} \sum_{\nu=0}^{a} z^{n} \operatorname{Res}_{j, \nu}\left(\frac{F(z)}{z}\right),
$$

where $C$ is a smooth Jordan curve enclosing the singularities of (7.2) and $\operatorname{Res}_{j, \nu}$ is the residue at the poles. The characteristic function

$$
g_{k}(z)=\left(z^{a}(z-b)\right)^{k}-b^{k}
$$

with restriction

$$
\left|\left\{\frac{(a+1)^{a+1}}{(a b)^{a}}\right\}^{k}\right|<1
$$

has exactly $(a+1) k$ distinct zeros $\xi_{j, \nu}$ for $j=0,1,2, \ldots, k-1$ and $\nu=0,1,2, \ldots, a$. A proof of this statement has been given by Sofo and Cerone [5]. From (7.2), then

$$
\begin{aligned}
f_{n} & =\sum_{j=0}^{k} \sum_{\nu=0}^{a} Q\left(\xi_{j, \nu}\right) \xi_{j, \nu}^{n}, \text { where } \\
Q\left(\xi_{j, \nu}\right) & =\lim _{z \rightarrow \xi_{j, \nu}}\left[\left(z-\xi_{j, \nu}\right) \frac{F(z)}{z}\right] \\
& =\frac{\xi_{j, \nu}}{k\left(\xi_{j, \nu}-b\right)^{k-1}\left((a+1) \xi_{j, \nu}-a b\right)} .
\end{aligned}
$$

From (7.3), (7.6) and (7.7)

$$
\begin{aligned}
& \sum_{r=0}^{[(n-k+1) /(a+1) k]}\left(\begin{array}{c}
n-a k r \\
k r+k-1
\end{array}\right) b^{n-a k r-k+1} \\
& =\sum_{j=0}^{k-1} \sum_{\nu=0}^{a} \frac{\xi_{j, \nu}^{n+1}}{k\left(\xi_{j, \nu}-b\right)^{k-1}\left((a+1) \xi_{j, \nu}-a b\right)} .
\end{aligned}
$$

If we let $\xi_{j, 0}, j=0,1,2, \ldots, k-1$ be the $k$ dominant zeros of (7.4) then we have the following theorem. 


\section{THE $k$ THEOREM}

THEOREM 1. Let

$$
\begin{gathered}
T_{r}(k, n, a, b)=\left(\begin{array}{c}
n-a k r \\
k r+k-1
\end{array}\right) b^{n-a k r-k+1} \text { and } \\
S(k, n, a, b)=\sum_{r=0}^{\infty} T_{r}(k, n, a, b),
\end{gathered}
$$

which is convergent for all values of $k, n, a$ and $b$ in the region of convergence (7.5). Then

$$
S(k, n, a, b)=\sum_{j=0}^{k-1} \frac{\xi_{j, 0}^{n+1}}{k\left(\xi_{j, 0}-b\right)^{k-1}\left((a+1) \xi_{j, 0}-a b\right)} .
$$

The series (7.8) with (8.3) gives us

$$
\begin{gathered}
\sum_{r=0}^{[(n-k+1) /(a+1) k]} T_{r}(k, n, a, b)+\sum_{r=[(n+a k+1) /(a+1) k]}^{\infty} T_{r}(k, n, a, b) \\
=\sum_{j=0}^{k-1} \frac{\xi_{j, 0}^{n+1}}{k\left(\xi_{j, 0}-b\right)^{k-1}\left((a+1) \xi_{j, 0}-a b\right)}
\end{gathered}
$$

and hence

$$
\sum_{r=[(n+a k+1) /(a+1) k]}^{\infty} T_{r}(k, n, a, b)=-\sum_{j=0}^{k-1} \sum_{\nu=1}^{a} \frac{\xi_{j, \nu}^{n+1}}{k\left(\xi_{j, \nu}-b\right)^{k-1}\left((a+1) \xi_{j, \nu}-a b\right)} .
$$

ProOF: The characteristic function (7.4) may be expressed as the product of factors

$$
g_{k}(z)=\prod_{j=0}^{k-1}\left(z^{a+1}-b z^{a}-b e^{(2 \pi i j) / k}\right)=\prod_{j=0}^{k-1} q_{j}(z) .
$$

For each of the $j$ factors in (8.4) we write

$$
F_{j}(z)=\frac{z^{a+1}}{z^{a+1}-b z^{a}-b e^{(2 \pi i j) / k}}
$$

for $j=0,1,2, \ldots, k-1$. The characteristic function $q_{i}(z)$ in (8.4) has exactly $a+1$ distinct zeros for each $j$, of which $\alpha_{j, 0}$ shall indicate the dominant zero, the one with the largest modulus, which may be complex. All the singularities in (8.5) are simple and therefore for each $j, F_{j}(z)$ has simple poles. Sofo and Cerone [6] have proved, from using (8.5) and evaluating its residue, that

$$
\sum_{r=0}^{\infty} e^{(2 \pi i j r) / k}\left(\begin{array}{c}
n-a r \\
r
\end{array}\right) b^{n-a r}=\frac{\alpha_{j, 0}^{n+1}}{(a+1) \alpha_{j, 0}-a b}
$$


for each $j=0,1,2, \ldots, k-1$. Note that (8.6) may in fact be a complex number. Summing (8.6) over $j$ gives

$$
\sum_{j=0}^{k-1} \sum_{r=0}^{\infty} e^{(2 \pi i j r) / k}\left(\begin{array}{c}
n-a r \\
r
\end{array}\right) b^{n-a r}=\sum_{j=0}^{k-1} \frac{\alpha_{j, 0}^{n+1}}{(a+1) \alpha_{j, 0}-a b} .
$$

Rescaling the left hand side by $r=\left(r^{*}+1\right) k$, (and then replacing $r^{*}$ by $r$ ) and changing the order of summation, results in

$$
\begin{aligned}
\sum_{r=-1}^{\infty} \sum_{j=0}^{k-1} e^{2 \pi i j(r+1)}\left(\begin{array}{c}
n-a k(r+1) \\
k(r+1)
\end{array}\right) b^{n-a k(r+1)} & =\sum_{j=0}^{k-1} \frac{\alpha_{j, 0}^{n+1}}{(a+1) \alpha_{j, 0}-a b}, \\
\sum_{r=0}^{\infty}\left(\begin{array}{c}
n-a k(r+1) \\
k(r+1)
\end{array}\right) b^{n-a k(r+1)} & =\sum_{j=0}^{k-1} \frac{\alpha_{j, 0}^{n+1}}{k\left((a+1) \alpha_{j, 0}-a b\right)}-b^{n}
\end{aligned}
$$

Now, make the substitution $n-a k=m$ in (8.7), giving upon simplification

$$
\sum_{r=0}^{\infty}\left(\begin{array}{c}
m-a k r \\
k r+k
\end{array}\right) b^{-a k r}=\sum_{j=0}^{k-1} \frac{b^{-m} \alpha_{j, 0}^{m+a k+1}}{k\left((a+1) \alpha_{j, 0}-a b\right)}-b^{a k} .
$$

Newton's forward difference formula of a function $h\left(x_{j}\right)=h_{j}$ at $x_{j}$ is defined as

$$
\Delta^{\rho} h_{j}=\Delta^{\rho-1} h_{j+1}-\Delta^{\rho-1} h_{j}, \quad \rho=1,2,3, \ldots .
$$

Taking the first difference of (8.8) with respect to $m$ results in, from the left hand side

$$
\sum_{r=0}^{\infty}\left(\begin{array}{c}
m+1-a k r \\
k r+k
\end{array}\right) b^{-a k r}-\sum_{r=0}^{\infty}\left(\begin{array}{c}
m-a k r \\
k r+k
\end{array}\right) b^{-a k r}=\sum_{r=0}^{\infty}\left(\begin{array}{c}
m-a k r \\
k r+k-1
\end{array}\right) b^{-a k r}
$$

Similarly, from the right hand side of (8.8)

$$
\sum_{j=0}^{k-1} \frac{\left(b^{-(m+1)} \alpha_{j, 0}^{m+a k+2}-b^{-m} \alpha_{j, 0}^{m+a k+1}\right)}{k A_{\alpha}}=\sum_{j=0}^{k-1} \frac{\left(b^{-m} \alpha_{j, 0}^{m+a k+1}\right)}{k A_{\alpha}} \cdot \frac{\left(\alpha_{j, 0}-b\right)}{b},
$$

where $A_{\alpha}=(a+1) \alpha_{j, 0}-a b$. From the characteristic function $q_{j}(z)$ in (8.4) $\alpha_{j, 0}^{a}\left(\alpha_{j, 0}-b\right)-$ $b e^{(2 \pi i j) / k}=0$, so we may write $(8.10)$ as

$$
\sum_{j=0}^{k-1} \frac{b^{-m+k-1} \alpha_{j, 0}^{m+1}}{k A_{\alpha}\left(\alpha_{j, 0}-b\right)^{k-1}}
$$

Combining with (8.9) gives, after simplification,

$$
\sum_{r=0}^{\infty}\left(\begin{array}{c}
m-a k r \\
k r+k-1
\end{array}\right) b^{m-a k r-k+1}=\sum_{j=0}^{k-1} \frac{\alpha_{j, 0}^{m+1}}{k\left(\alpha_{j, 0}-b\right)^{k-1}\left((a+1) \alpha_{j, 0}-a b\right)} .
$$

Since the dominant zeros $\alpha_{j, 0}, j=0,1,2, \ldots, k-1$ of $q_{j}(z)$ are the same as the dominant zeros $\xi_{j, 0}$ of $g_{k}(z)$ in (7.4) (a proof of this statement has been given by Sofo and Cerone [5]), then upon renaming $m$ as $n$ in (8.11), Theorem 1 is proved since (8.11) and (8.3) are identical. Note that putting $k=1$ in (8.3) yields result (2.6) for $R=1$. 


\section{Numerical Results and Special Cases}

In the following numerical results, the dominant zeros $\xi_{j, 0}$ are evaluated from $g_{k}(z)$ in (7.4). It may also be noted that for $k \geqslant 3$ the dominant zeros occur in complex conjugate pairs. The numerical results are given to four significant digits.

\begin{tabular}{|c|c|c|c|c|c|}
\hline$k$ & $n$ & $a$ & $b$ & $\xi_{j, 0}$ & identity (8.3) \\
\hline 2 & 3 & 2 & 10 & $\left.\begin{array}{l}\xi_{0,0}=9.8979 \\
\xi_{1,0}=10.0981\end{array}\right\}$ & 299.9899 \\
\hline 3 & 3 & 1 & -10 & $\begin{array}{l}\xi_{0,0}=-8.8730 \\
\xi_{1,0}=-10.5329+0.7826 i \\
\xi_{2,0}=-10.5329-0.7826 i\end{array}$ & -30.0005 \\
\hline 3 & 3 & 2 & 10 & $\begin{array}{l}\xi_{0,0}=10.0981 \\
\xi_{1,0}=9.9511+0.0883 i \\
\xi_{2,0}=9.9511+0.0883 i\end{array}$ & 29.9998 \\
\hline
\end{tabular}

The degenerate case, $a=0$, of (8.3) yields the result

$$
\begin{aligned}
\sum_{r=0}^{[(m-k+1) / k]}\left(\begin{array}{c}
m \\
k r+k-1
\end{array}\right) & =\sum_{j=0}^{k-1} \frac{\left(1+e^{(2 \pi i j) / k}\right)^{m}}{k e^{(2 \pi i j(k-1)) / k}} \\
& =\frac{2^{m}}{k} \sum_{j=0}^{k-1} e^{(\pi i j(m+2)) / k} \cos ^{m}\left(\frac{\pi j}{k}\right),
\end{aligned}
$$

and for $k=4$, we have

$$
\sum_{r=0}^{[(m-3) / 4]}\left(\begin{array}{c}
m \\
4 r+3
\end{array}\right)=\frac{1}{4}\left(2^{m}-2^{(m / 2)+1} \sin \frac{m \pi}{4}\right) .
$$

By using the $W Z$ pairs method of Wilf and Zeilberger, we find that rational function proof certificates $V_{k}(m, r)$, for $k=1$ and 2 of $(9.1)$ are

$$
V_{1}(m, r)=\frac{r}{2(r-1-m)} \text { and } V_{2}(m, r)=\frac{(r-1)(2 r-1)}{m(2 r-2-m)} \text {. }
$$




\section{The Hypergeometric Connection}

Consider the term $T_{r}(k, n, a, b)$ of $(8.1)$ with $T_{0}(k, n, a, b)=\left(\begin{array}{c}n \\ k-1\end{array}\right) b^{n-k+1}$. The ratio of consecutive terms

$$
\begin{aligned}
& \frac{T_{r+1}(k, n, a, b)}{T_{r}(k, n, a, b)} \\
& \quad=\frac{\prod_{j=0}^{(a+1) k-1}(r+((j+k-n-1) /(a+1)) k)}{(r+1) \prod_{j=0}^{a k-1}(r+((j-n) /(a k))) \prod_{j=0}^{k-2}(r+((2 k-j-1) / k))} s(a, b, k)
\end{aligned}
$$

is a rational function in $r$ and therefore the series $S(k, n, a, b)$ of $(8.2)$ may be expressed as a generalised hypergeometric function

$$
\left.\begin{array}{l}
T_{0(a+1) k} F_{(a+1) k-1} \\
{\left[\begin{array}{l}
\frac{k-n-1}{(a+1) k}, \frac{k-n}{(a+1) k}, \ldots, \frac{k-n-1+(a+1) k-1}{(a+1) k} \\
-\frac{n}{a k}, \frac{1-n}{a k}, \ldots, \frac{a k-1-n}{a k}, \frac{2 k-1}{k}, \frac{2 k-2}{k}, \ldots, \frac{2 k-1-(k-2)}{k}
\end{array} \mid s(a, b, k)\right.}
\end{array}\right],
$$

where

$$
s(a, b, k)=\left(-\frac{(a+1)^{a+1}}{(a b)^{a}}\right)^{k} .
$$

A simple example shows that, from (8.2) and (8.3) for $k=2, a=1, b=x^{-1}$ and $n=-\alpha \in \mathbb{R}$, we have two distinct dominant zeros of $(7.4), 2 x \xi_{0,0}=1+\sqrt{1+4 x}$, $2 x \xi_{1,0}=1+\sqrt{1-4 x}$ and therefore

$$
\begin{aligned}
\sum_{r=0}^{\infty}\left(\begin{array}{c}
4 r+\alpha \\
2 r+1
\end{array}\right) x^{2 r} & =\sum_{j=0}^{1} \frac{\xi_{j, 0}^{1-\alpha}}{2\left(x \xi_{j, 0}+1\right)\left(2 x \xi_{j, 0}-1\right)} \\
& =\alpha_{4} F_{3}\left[\begin{array}{c}
\frac{\alpha+1}{4}, \frac{\alpha+2}{4}, \frac{\alpha+3}{4}, \frac{\alpha+4}{4} \\
\frac{\alpha}{2}, \frac{\alpha+1}{2}, \frac{3}{2}
\end{array} \mid 16 x^{2}\right]
\end{aligned}
$$

Specifically with $\alpha=1$,

$$
\begin{aligned}
\sum_{r=0}^{\infty}\left(\begin{array}{c}
4 r+1 \\
2 r+1
\end{array}\right) x^{2 r} & ={ }_{4} F_{3}\left[\begin{array}{c}
\frac{1}{2}, \frac{3}{4}, 1, \frac{5}{4} \mid 16 x^{2} \\
\frac{1}{2}, 1, \frac{3}{2} \mid
\end{array}\right] \\
& =\frac{\sqrt{1-4 x}+\sqrt{1+4 x}-2}{(\sqrt{1+4 x}-(1+4 x))(\sqrt{1-4 x}-(1-4 x))} \\
& =\frac{1}{4 x}\left(\frac{1}{\sqrt{1-4 x}}-\frac{1}{\sqrt{1+4 x}}\right)=\sum_{r=0}^{\infty}\left(\begin{array}{c}
4 r+1 \\
2 r
\end{array}\right) x^{2 r}
\end{aligned}
$$

All the above identities may be differentiated and integrated to produce more results. 


\section{Non-Zero Forcing Terms}

If we consider system (7.1) with all initial conditions at rest and with a forcing term of the form $w_{n}=\left(\begin{array}{c}n \\ m-1\end{array}\right) b^{n-m+1}$ for $m$ integer and follow the procedure of the previous section, we obtain the identity

$$
\begin{aligned}
\sum_{r=0}^{\infty}\left(\begin{array}{c}
r+R-1 \\
r
\end{array}\right)\left(\begin{array}{c}
n-a k r \\
k r+R k+m-1
\end{array}\right) b^{-k a r-k R} & \\
=\sum_{\nu=0}^{m-1} P_{m, \nu}(b)\left(\begin{array}{c}
n \\
m-1-\nu
\end{array}\right) b^{\nu} & \\
& \quad+\sum_{\mu=0}^{R-1} \sum_{j=0}^{k-1} Q_{R, \mu}\left(\xi_{j, 0}\right) b^{m-n-1}\left(\begin{array}{c}
n \\
R-1-\mu
\end{array}\right) \xi_{j, 0}^{n-R+1+\mu}
\end{aligned}
$$

in the region of convergence (7.5) where

$$
\begin{aligned}
\nu ! P_{m, \nu}(b) & =\lim _{z \rightarrow b}\left[\frac{d^{\nu}}{d z^{\nu}}\left\{(z-b)^{m} \frac{F(z)}{z}\right\}\right], \\
\mu ! Q_{R, \mu}\left(\xi_{j, 0}\right) & =\lim _{x \rightarrow \xi_{j, 0}}\left[\frac{d^{\mu}}{d z^{\mu}}\left\{\left(z-\xi_{j, 0}\right)^{R} \frac{F(z)}{z}\right\}\right]
\end{aligned}
$$

and

$$
F(z)=\frac{z^{a k R+1}}{(z-b)^{m}\left(\left(z^{a}(z-b)\right)^{k}-b^{k}\right)^{R}} .
$$

For $R=1, k=1, n=-\alpha \in \mathbb{R}$ and $m=2$, from (11.1) we have

$$
\begin{aligned}
& \sum_{r=0}^{\infty}(-1)^{r}\left(\begin{array}{c}
(a+1) r+\alpha+1 \\
r+2
\end{array}\right)\left(\frac{1}{b^{a}}\right)^{r} \\
&=(\alpha-a) b^{a}-b^{2 a}+\frac{b^{2+\alpha} \xi_{0,0}^{1-\alpha}}{\left(\xi_{0,0}-b\right)^{2}\left((a+1) \xi_{0,0}-a b\right)}
\end{aligned}
$$

where $\xi_{0,0}$ is the dominant zero which satisfies (7.4), and specifically for $(a, \alpha)=(1, \alpha)$, $2 \xi_{0,0}=b+\sqrt{b^{2}+4 b}$, so that

$$
\sum_{r=0}^{\infty}(-1)^{r}\left(\begin{array}{c}
2 r+\alpha+1 \\
r+2
\end{array}\right)\left(\frac{1}{b}\right)^{r}=(\alpha-1) b-b^{2}+\frac{b(2 b)^{\alpha}\left(b+\sqrt{b^{2}+4 b}\right)^{1-\alpha}}{(b+2) \sqrt{b^{2}+4 b}-\left(b^{2}+4 b\right)} .
$$

Many other identities of this form may be obtained by various manipulations. One such result is

$$
\sum_{r=0}^{\infty}\left(\begin{array}{c}
r+1 \\
r
\end{array}\right)\left(\begin{array}{c}
4 r+4 \\
2 r+2
\end{array}\right) x^{-2 r}=\frac{x}{2}\left(\left(\frac{x}{x-4}\right)^{3 / 2}-\left(\frac{x}{x+4}\right)^{3 / 2}\right)
$$




\section{Conclusion}

A method for the summation of infinite series has been described and an extension of Jensen's form given. The generation of an infinite sum was achieved by the application of $Z$ transforms and the closed form representation was proved by an induction argument. The method described in this paper may be easily extended to handle, for example, systems with multiple delays. Sofo and Cerone [7] have also obtained other identities by considering arbitrary order-forced differential-difference dynamical systems.

\section{REFERENCES}

[1] M. Abramowitz and I. Stegun, Handbook of mathematical functions (Dover Publications Inc., New York, 1970).

[2] J.W. Cohen, The single server queue, (Revised edition) (North-Holland Publishing Company, Amsterdam, 1982).

[3] J.L.W.V. Jensen, 'Sur une identite d'Abel et sur d'autres formules analogues', Acta Math. 26 (1902), 307-313.

[4] M. Petkovšek, H.S. Wilf and D. Zeilberger, $A=B$ (A.K. Peters, Wellesley, M.A., 1996).

[5] A. Sofo and P. Cerone, Summation of series of binomial variation, Report 86 Math 13 (Victoria University of Technology, 1997).

[6] A. Sofo and P. Cerone, 'On a Fibonacci related series', Fibonacci Quart. 36 (1998), 211-215.

[7] A. Sofo and P. Cerone, 'Generalisation of a waiting-time relation', J. Math. Anal. Appl. 214 (1997), 191-206.

[8] A. Sofo and P. Cerone, 'Generalisation of Euler's identity', Bull. Austral. Math. Soc. 58 (1998), 359-371.

[9] H.S. Wilf, Generatingfunctionology (Academic Press Inc., New York, 1994).

School of Communications and Informatics

Victoria University of Technology

P.O. Box 14428

Melbourne City MC

Victoria 8001

Australia

e-mail: anthony.sofo@vu.edu.au

http://sci.vu.edu.au/staft/anthonys.html 